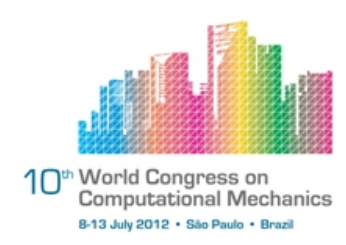

\title{
Evolutionary Topology Optimization for Fluid-structure Interaction Problems and Natural Frequency Maximization
}

\author{
R. Picelli ${ }^{1}$, W. M. Vicente ${ }^{1}$, R. Pavanello ${ }^{1}$ \\ ${ }^{1}$ Department of Computational Mechanics, Faculty of Mechanical Engineering at the State \\ University of Campinas (picelli@fem.unicamp.br)
}

\begin{abstract}
Multiphysics systems including dynamic fluid-structure interaction problems have hardly been studied in several fields of mechanical engineering. Among others, we can cite the researches in vibrations of submerged structures and the design of poroacoustic absorbing systems. Structural Topology Optimization can be applied in this class of problems in order to obtain new materials and structures. In this paper, it is presented the topology optimization based on volume constraints and natural frequency maximization of fluid-structure interaction problems. The method used in this work is the Bi-directional Evolutionary Structural Optimization (BESO), which consists in a successive elimination and replacement of elements in the design domain. This domain is defined initially and through a sensitivity analysis of the structure's eigenvalue solution, the evolutionary algorithm remove or add solid elements. The aim of this work is to propose a new version of the BESO method applied to fluid-structure interaction systems. We consider the case of free vibration of structures attached to a fixed fluid domain. Numerical results show that the BESO method can be applied to this kind of multiphysics problem.
\end{abstract}

Keywords: Fluid-structure interaction, Topology Optimization, BESO method.

\section{INTRODUCTION}

Since its introduction more than two decades ago [1], Structural Topology Optimization method for continuum structures [2] has gained in popularity and now is used daily as a design tool in industry and academy. In contrast to size and shape optimization, topology optimization can be used to change the topologies inside design domains and to find optimal layouts without the need for an initial given topology [3]. Furthermore, commercial topology optimization tools have been developed based on special Finite Element Method (FEM) solvers or have been added in standard commercial packages.

The applications of topology optimization methods have been extended to many cases, e.g. material design [4], microelectromechanical systems (MEMs) design [5], synthesis of acoustic absorbers [6] and others. The most common problems solved by commercial codes concern stiffness and natural frequency maximization, both with volume fraction constraints. 
Although these procedures have reached a satisfactory level of maturity, there are still many topology optimization problems open to research or less-than-satisfactorily resolved issues [7]. A major group consists of multiphysical problems. Commercial FEM packages often contain solvers for multiphysics problems, however they do not enable optimization. A particular class of multiphysics problems involves fluid-structure interaction (FSI), which is the main topic of this work.

Multiphysical problems with fluid-structure interaction are very common in structural engineering. Only a few authors have studied the topology optimization of FSI coupled systems. Between them, we can cite Maute and Allen [8] who presented the topology optimization of aeronautic structures considering the aeroelastic coupling with the classical material interpolation method 'SIMP' (Solid Isotropic Material with Penalization) [9]. Also, the contribution of Sigmund and Clausen [7] stands out, who proposed a mixed formulation in the SIMP method to optimize structures under external pressure loads problems.

More recently, Hamidian and Seyedpoor [10] presented the shape optimization of arch dams considering fluid-structure interaction. Kreissl et. al [11] optimized microfluidic systems considering non-linearity and the LBM hydrodynamics using topology optimization. Yoon [3] introduced a monolithic formulation for topology optimization of FSI coupled problems. Recent publications show the current scientific effort devoted on this topic.

Among the current optimization methods, the Bi-directional Evolutionary Structural Optimization (BESO) was chosen in this work for FSI problems analysis, since the method is almost not explored in this area. The technique so called Evolutionary Structural Optimization (ESO) was first introduced in the 90's with Xie and Steven [12]. With the finite element analysis, the ESO method was initially proposed as a gradual removal of inefficient material from the design domain until the remaining structure converges to the optimum topology. Material elimination is done after a sensitivity analysis. However, it is questionable that eliminated elements can never return to the design domain, since the sensitivity analysis is carried out only for the solid elements in the structure. Thus, a later development of this method was called Bi-directional ESO (BESO), in which elements are also added in void positions near to the elements with the highest sensitivity numbers [13]. In this case, the sensitivity number is a local index and represent the sensitivity of each element with respect to the objective function. Studies with the BESO method have recently been published presenting convergent and mesh independent solutions for stiffness maximization [14], for natural frequency maximization [15] and others. Critical analysis of ESO-based methods are found in [16], while a later review about the ESO/BESO methods is found in [17] and a book is also available [18]. In multiphysics problems, the ESO/BESO methods lack of methodologies and publications, especially in fluid-structure coupled systems.

Herewith it is proposed to combine the evolutionary topology optimization techniques with multiphysics problems involving fluid-structure interaction. This work focuses on modal analysis where the aim is to increase the natural frequencies of the coupled system. Frequency optimization is of great importance in many engineering fields and the modal analysis is applicable to a wide range of fluid-structure interaction problems. The possibility of removing and adding material systematically with the evolutionary method might be a helpful procedure to explicitly define the fluid-structure interfaces. However, only cases with immovable 
interfaces will be considered in this paper.

The paper is organized as follows: Section 2 presents the governing equations and the finite element model for the fluid-structure interaction coupled system. In Section 3, the topology optimization problem for frequency maximization is formulated and the sensitivity number is derived. Section 4 discusses the implementation matters and the basics of the BESO method. Section 5 concludes the paper.

\section{FLUID-STRUCTURE INTERACTION: GOVERNING EQUATIONS AND FINITE ELEMENT MODEL}

Herein, the analyzed systems are limited to a flexible structure in contact with an enclosed acoustic fluid, where the responses of the structure are significantly affected by the fluid. For this fluid-structure system, the structure can be described by the differential equation of motion for a continuum body assuming small deformations and the fluid by the acoustic wave equation. For the standard approach, the governing equations for the fluid and structural domains as well as the coupling boundary conditions are defined as follows.

\subsection{Helmholtz equation}

In this paper, the fluid is considered inviscid, irrotational and only under small translations conditions. The governing equation for the pressure in a nonhomogeneous acoustic medium is described by the Helmholtz equation

$$
\nabla^{2} p_{f}+\frac{\omega^{2}}{c_{f}^{2}} \frac{\partial^{2} p_{f}}{\partial t^{2}}=0 \quad\left(k=\frac{\omega}{c_{f}}\right) \quad \Omega_{f}
$$

where $p_{f}$ is the pressure in the analysis domain $\Omega_{f}, c_{f}$ is the local speed of sound, $\omega$ is the angular frequency and $k$ the wave number. The pressure field is obtained by solving the Helmholtz equation imposing proper boundary conditions. In this paper, it is considered the following boundary conditions:

$$
\begin{gathered}
p_{f}=p_{0} \\
\mathbf{n} \nabla p_{f}=0
\end{gathered}
$$

representing the pressure boundary condition (Eq. 2) and the hard wall condition (Eq. 3), where $p_{0}$ is the pressure input and $\mathbf{n}$ is the outward unit normal to the fluid.

\subsection{Linear elasticity}

The linear structural analysis can be described by the elasticity equation

$$
\tilde{\nabla}^{T} \sigma_{s}+\mathbf{b}_{s}=\rho_{s} \frac{\partial^{2} \mathbf{u}_{s}}{\partial t^{2}} \quad \Omega_{s}
$$

where $\sigma_{s}$ is the stress tensor, $\mathbf{b}_{s}$ are the body forces and $\mathbf{u}_{s}$ is the displacements field. Boundary conditions are applied as follows: 


$$
\begin{array}{cc}
\mathbf{n}_{s} \sigma=\mathbf{f}^{S_{b}} & S_{b} \\
\mathbf{u}_{s}=\mathbf{u}_{s}^{S_{u}} & S_{u}
\end{array}
$$

representing the Neumann (Eq. 5) and Dirichlet (Eq. 6) boundary conditions, where $f^{S_{b}}$ is the surface traction on $S_{b}, \mathbf{u}_{s}^{S_{u}}$ is the prescribed displacements on $S_{u}$ and $\mathbf{n}_{s}$ is the outward unit normal to the solid medium.

\subsection{The coupled fluid-structure system}

At the interface $\partial \Omega_{s f}$ between the structural and fluid domains, the fluid and the structure move together in the normal direction of the boundary. The normal vector $\mathbf{n}=\mathbf{n}_{f}=-\mathbf{n}_{s}$ can be used in order to obtain the displacement boundary condition

$$
\left.\mathbf{u}_{s} \mathbf{n}\right|_{\partial \Omega_{s f}}=\left.\mathbf{u}_{f} \mathbf{n}\right|_{\partial \Omega_{s f}}
$$

and the continuity in pressure

$$
\left.\sigma_{s}\right|_{n}=-p
$$

With relations derived from the governing equations and the previous coupling conditions, the interface forces may be obtained. The force acting on the structure provided by the fluid pressure is

$$
\mathbf{f}_{f}=\int_{\Omega_{s f}} \mathbf{N}_{s}^{T} \mathbf{n} \mathbf{N}_{f} d s \mathbf{p}_{\mathbf{f}}
$$

and the force acting on the fluid domain can be expressed in structural acceleration

$$
\mathbf{f}_{s}=-\rho_{f} \int_{\Omega_{s f}} \mathbf{N}_{f}^{T} \mathbf{n} \mathbf{N}_{s} d s \ddot{\mathbf{u}}_{\mathbf{s}}
$$

where $\rho_{f}$ is the static density of the fluid and $\mathbf{N}$ contains the finite element shape functions for the interface.

The introduction of a spatial coupling matrix

$$
\mathbf{L}_{s f}=\int_{\Omega_{s f}} \mathbf{N}_{s}^{T} \mathbf{n} \mathbf{N}_{f} d s
$$

allows the coupling force to be written as

$$
\mathbf{f}_{f}=\mathbf{L}_{s f} \mathbf{p}_{f}
$$

and

$$
\mathbf{f}_{s}=-\rho_{f} \mathbf{L}_{s f}^{T} \ddot{\mathbf{u}}_{s}
$$

Thus, the fluid-structure problem can then be described by an unsymmetrical system of equations 


$$
\left[\begin{array}{cc}
\mathbf{M}_{s} & \mathbf{0} \\
\rho_{f} \mathbf{L}_{s f}^{T} & \mathbf{M}_{f}
\end{array}\right]\left[\begin{array}{l}
\ddot{\mathbf{u}}_{s} \\
\ddot{\mathbf{p}}_{f}
\end{array}\right]+\left[\begin{array}{cc}
\mathbf{K}_{s} & -\mathbf{L}_{s f} \\
\mathbf{0} & \mathbf{K}_{f}
\end{array}\right]\left[\begin{array}{l}
\mathbf{u}_{s} \\
\mathbf{p}_{f}
\end{array}\right]=\left[\begin{array}{c}
\mathbf{f}_{s} \\
\mathbf{f}_{f}
\end{array}\right]
$$

where $\mathbf{f}_{s}$ and $\mathbf{f}_{f}$ are the load vectors for both domains.

In the multiphysics coupling analysis, the fluid analysis provides pressure loads to the structural analysis, and the structural analysis provides accelerations to the fluid analysis. These finite element model has been used to calculate responses of acoustic-structure interaction problems. For details and the derivations of the coupling integrals as well as the governing equations, the reader is referred to [19].

\section{TOPOLOGY OPTIMIZATION PROBLEM AND THE SENSIVITY ANALYSIS}

\subsection{Topology optimization problem}

In finite element analysis the following eigenproblem is solved to describe the dynamic behavior in free vibration of a structure:

$$
\left(\mathbf{K}-\omega_{i}^{2} \mathbf{M}\right) \Phi_{\mathbf{i}}=0
$$

If fluid-structure interaction is considered, $\mathbf{K}$ and $\mathbf{M}$ are, respectively, the global stiffness and mass matrices including the structural and fluid elemental matrices and the coupling integrals described in the previous section. $\omega_{i}$ is the $i$ th natural frequency and $\Phi_{i}$ is the corresponding eigenmode.

Premultiplying equation 15 with the transposed eigenvector, $\Phi_{i}^{T}$, the eigenvalue $\omega_{i}^{2}$ can be related with $\Phi_{i}$ by

$$
\omega_{i}^{2}=\frac{\Phi_{i}^{T} \mathbf{K} \Phi_{i}}{\Phi_{i}^{T} \mathbf{M} \Phi_{i}}
$$

which is called the Rayleigh quotient.

In this paper, the topology optimization problem for maximization of the $i$ th natural frequency of a fluid-structural vibrating system is considered. As pointed out earlier, this work discusses the analysis of a fluid-structure system with immovable interface. Thus, the design domain is restricted only to the structural region with responses influenced by the enclosed fluid. Then, for a solid-void design, the discrete optimization problem is enunciated as

$$
\begin{array}{cc}
\text { Maximize } & \omega_{i} \\
\text { Subject to: } & V_{f}-\sum_{i=1}^{n} V_{i} x_{i}=0 \\
& x_{i}=x_{\text {min }} \text { or } 1
\end{array}
$$

where $V_{i}$ is the volume of an individual element, $V_{f}$ the prescribed total structural volume and $n$ is the total number of elements in the system. The binary design variable $x_{i}$ declares the presence of a completely solid element (1) or the density of a void element with a small value of $x_{\min }$ (e.g. $10^{-4}$ ). It is possible to note that maximizing the $i$ th natural frequency is the same that maximizing the eigenvalue $\omega_{i}^{2}$. 


\subsection{Material interpolation scheme and sensitivity numbers}

The popular material interpolation function so-called SIMP (Simplified Isotropic Material with Penalization) demonstrated to be not viable for vibrating problems since a high ratio between the penalization on mass and stiffness causes artificial vibration modes in lowdensity regions [20]. For the soft-kill BESO method developed by Huang et al. [15], to keep the ratio between mass and stiffness constant when $x_{i}=x_{\min }$ it is required that

$$
\begin{aligned}
\rho\left(x_{\text {min }}\right) & =x_{\text {min }} \rho^{1} \\
E\left(x_{\text {min }}\right) & =x_{\text {min }} E^{1}
\end{aligned}
$$

where $\rho^{1}$ and $E^{1}$ are the density and Young's modulus of the solid material. The proposed material interpolation scheme is expressed by

$$
\begin{gathered}
\rho\left(x_{\text {min }}\right)=x_{i} \rho^{1} \\
E\left(x_{i}\right)=\left[\frac{x_{\min }-x_{\min }^{p}}{1-x_{\min }^{p}}\left(1-x_{i}^{p}\right)+x_{i}^{p}\right] E^{1} \quad\left(0<x_{\min } \leq x_{i} \leq 1\right)
\end{gathered}
$$

Thus, the derivatives of the global mass and stiffness matrices may be evaluated by

$$
\begin{gathered}
\frac{\partial \mathbf{M}}{\partial x_{i}}=\mathbf{M}_{i}^{1} \\
\frac{\partial \mathbf{K}}{\partial x_{i}}=\frac{1-x_{\text {min }}}{1-x_{\text {min }}^{p}} p x_{i}^{p-1} \mathbf{K}_{i}^{1}
\end{gathered}
$$

where $\mathbf{M}_{i}^{1}$ and $\mathbf{K}_{i}^{1}$ are the elemental mass and stiffness matrices of the solid element. It is reasonable to mention that once the fluid-structure interface does not change in this work, the coupling matrices are constant and do not appear in the derivatives.

The derivative with respect to the design variable $x_{i}$ is the sensitivity of our objective function (Eq. 16). This derivative is expressed by

$$
\frac{d \omega_{i}}{d x_{i}}=\frac{1}{2 \omega_{i} \boldsymbol{\Phi}_{i}^{T} \mathbf{M} \boldsymbol{\Phi}_{i}}\left[2 \frac{\partial \boldsymbol{\Phi}_{i}^{T}}{\partial x_{i}}\left(\mathbf{K}-\omega_{i}^{2} \mathbf{M}\right) \boldsymbol{\Phi}_{i}+\boldsymbol{\Phi}_{i}^{T}\left(\frac{\partial \mathbf{K}}{\partial x_{i}}-\omega_{i}^{2} \frac{\partial \mathbf{M}}{\partial x_{i}}\right) \boldsymbol{\Phi}_{i}\right]
$$

with the equation 15 , the derivative may be simplified as

$$
\frac{d \omega_{i}}{d x_{i}}=\frac{1}{2 \omega_{i} \boldsymbol{\Phi}_{i}^{T} \mathbf{M} \boldsymbol{\Phi}_{i}}\left[\boldsymbol{\Phi}_{i}^{T}\left(\frac{\partial \mathbf{K}}{\partial x_{i}}-\omega_{i}^{2} \frac{\partial \mathbf{M}}{\partial x_{i}}\right) \boldsymbol{\Phi}_{i}\right]
$$

With the derivatives of $\mathbf{K}$ and $\mathbf{M}$ global matrices from equations 20 and 21 and considering that the eigenvector is mass-normalized $\left(\boldsymbol{\Phi}_{i}^{T} \mathbf{M} \boldsymbol{\Phi}_{i}=1\right)$, the sensitivity for the $i$ th natural frequency is

$$
\frac{d \omega_{i}}{d x_{i}}=\frac{1}{2 \omega_{i}} \boldsymbol{\Phi}_{i}^{T}\left(\frac{1-x_{\min }}{1-x_{\min }^{p}} p x_{i}^{p-1} \mathbf{K}_{i}^{1}-\omega_{i}^{2} \mathbf{M}_{i}^{1}\right) \boldsymbol{\Phi}_{i}
$$

For the evolutionary topology optimization, the discrete design variables $\left(x_{\min }\right.$ and 1$)$ declare the element state. Again, even though our structure is enclosed to a fluid domain, our design domain will consist to a solid-void region. Thus, the sensitivity numbers to rank the elemental sensitivities are identical to the ones proposed by Huang et al. [15] as 


$$
\begin{aligned}
\alpha_{i}=\frac{1}{p} \frac{d \omega_{i}}{d x_{i}} & =\frac{1}{2 \omega_{i}} \boldsymbol{\Phi}_{i}^{T}\left(\frac{1-x_{\min }}{1-x_{\min }^{p}} \mathbf{K}_{i}^{1}-\frac{\omega_{i}^{2}}{p} \mathbf{M}_{i}^{1}\right) \boldsymbol{\Phi}_{i} & & x_{i}=1 \\
& =\frac{1}{2 \omega_{i}} \boldsymbol{\Phi}_{i}^{T}\left(\frac{x_{\min }^{p-1}-x_{\min }^{p}}{1-x_{\min }^{p}} \mathbf{K}_{i}^{1}-\frac{\omega_{i}^{2}}{p} \mathbf{M}_{i}^{1}\right) \boldsymbol{\Phi}_{i} & & x_{i}=x_{\min }
\end{aligned}
$$

or when $x_{\min }$ tends to 0

$$
\begin{aligned}
\alpha_{i}=\frac{1}{p} \frac{d \omega_{i}}{d x_{i}} & =\frac{1}{2 \omega_{i}} \boldsymbol{\Phi}_{i}^{T}\left(\mathbf{K}_{i}^{1}-\frac{\omega_{i}^{2}}{p} \mathbf{M}_{i}^{1}\right) \boldsymbol{\Phi}_{i} & & x_{i}=1 \\
& =-\frac{\omega_{i}}{p} \boldsymbol{\Phi}_{i}^{T} \mathbf{M}_{i}^{1} \boldsymbol{\Phi}_{i} & & x_{i}=x_{\text {min }}
\end{aligned}
$$

It is important to point out that also when $x_{\min }$ tends to $0, x_{i}=x_{\min }$ for a void element, which indicates that the element is not completely eliminated from the design domain as proposed for the soft-kill BESO method [15,21].

\section{IMPLEMENTATION MATTERS}

Originally, the evolutionary topology optimization was based on a successive elimination of material from the initial design domain [12]. One of the last great developments in the ESO-based methods was the bi-directional version, the BESO method [14]. The BESO method allows material to be simultaneously removed and added in the domain until the volume constraint and a convergence criterion are satisfied. To rank all the elements, the sensitivity numbers from equation 26 are evaluated for each element. A mesh-independency filter is applied all over the mesh by averaging the elemental sensitivity number with its neighbouring elements. This filter scheme is similar to that used in [22] and it is a heuristic technique. First of all, nodal sensivity numbers $\alpha_{j}^{n}$ are created by averaging the elemental sensitivity numbers of the jth connected elements. These nodal sensitivity numbers must be converted back into elemental sensitivities by projecting a sub-domain $\Psi$ with length scale $r_{\min }$ and center in the $i$ th element. All the nodes inside $\Psi$ must have their nodal sensitivity numbers averaged back to the $i$ th elemental level as follows:

$$
\alpha_{i}=\frac{\sum_{j=1}^{M} w\left(r_{i j}\right) \alpha_{j}^{n}}{\sum_{j=1}^{M} w\left(r_{i j}\right)}
$$

where $r_{i j}$ is the distance between the node $j$ and the center of the element $i, M$ is the total number of nodes in the structure and $w\left(r_{i j}\right)$ is a weight factor that values $r_{\min }-r_{i j}$ for nodes inside $\Psi$ and 0 for outside nodes. Huang et al. [14] also showed that the above sensitivity numbers should be averaged with its previous iteration numbers. It demonstrated to be a effective way to avoid convergence problems.

For each iteration, a target volume $V_{k+1}$ is defined as

$$
V_{k+1}=V_{k}(1 \pm E R)
$$

where $E R$ is the evolutionary ratio and $k$ the number of the iteration. $E R$ is the percentage of the initial design domain volume and increases or decreases $V_{k+1}$ towards a desired final volume $V_{f}$. The addition volume is restricted to an addition ratio $A R_{\max }$, which declares the maximum allowable addition volume per iteration. Each target volume defines the number of 
elements that the structure must have in the iteration $k+1$. It designates a threshold where the elements with higher sensitivity numbers should change from $x_{\min }$ to 1 and from 1 to $x_{\min }$ for those with lower sensitivity numbers. Hence, the filter scheme plays an important role. The projection scheme allows void elements $\left(x_{\text {min }}\right)$ near highly solicited solid regions to have a bigger sensitivity number and return to solid (1) condition. It also controls the size of the members in the structure related to the $r_{\min }$ parameter. It is important to point out that for all the elements of our fixed fluid domain a big sensitivity number is given. Thus, with the projection scheme, all the solid elements near the fluid region will have a big averaged sensitivity number. Therefore, these solid elements will never be eliminated from the domain, which in practice ensures a constant and immovable fluid-structure interface.

Once the prescribed final volume is achieved, the target volume remains constant as $V_{f}$. The algorithm evolves until a convergence criterion with a predefined tolerance $\tau$ is satisfied. All the details about the BESO implementation may be referred to the paper [14].

\section{NUMERICAL RESULTS}

\subsection{Beam with clamped ends}

In this first example, we want to investigate the influence of a fluid domain in the eigenmodes of a structure and in the topology optimization. A beam with clamped ends was chosen as the design domain for natural frequencies maximization in a fluid-structure model, as seen in Fig. 1. Hard-wall condition and a pressure input were imposed to the fluid part.

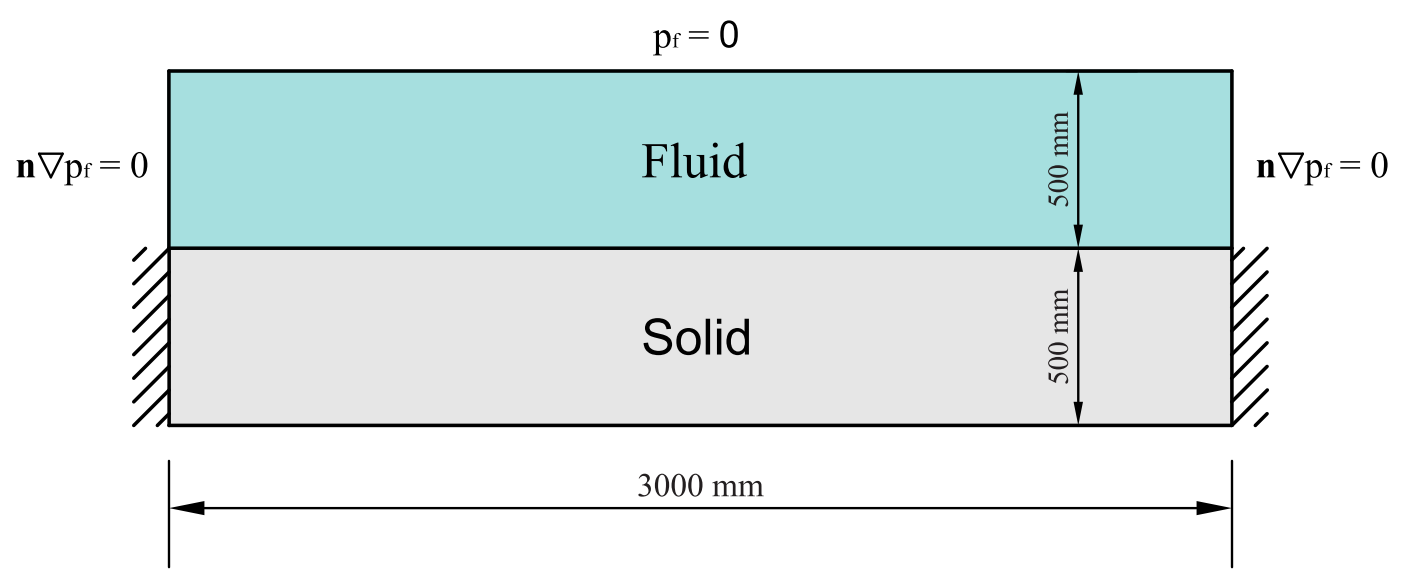

Figure 1. Fluid-structure model.

The beam with dimensions $3000 \times 500 \mathrm{~mm}$ is equally divided into $120 \times 20$ fournode plane stress elements. The material is considered with Young's modulus $E=70 \mathrm{GPa}$, Poisson's ratio $\nu=0.3$ and mass density $\rho_{s}=2700 \mathrm{~kg} / \mathrm{m}^{3}$. The fluid domain has the same dimensions and discretization of the solid domain, considering its mass density $\rho_{f}=1000$ $\mathrm{kg} / \mathrm{m}^{3}$ and the speed of sound in the fluid $c=1450 \mathrm{~m} / \mathrm{s}$. The whole model has unitary thickness.

In order to analyze the eigenmodes of both solid and fluid domain and the interaction between them, the eigenproblem was solved separated for only the solid domain, only the fluid domain and the complete coupled system. Table 1 presents the first four natural frequencies and eigenmodes for these models. 
Table 1. First four natural frequencies and eigenmodes for the solid domain, the fluid domain and the coupled fluid-structure model.

\begin{tabular}{|c|c|c|c|c|c|c|}
\hline & \multicolumn{2}{|c|}{ Solid domain } & \multicolumn{2}{|c|}{ Fluid domain } & \multicolumn{2}{|c|}{ Coupled system } \\
\hline & $\omega(\mathrm{Hz})$ & Eigenmode & $\omega(\mathrm{Hz})$ & Eigenmode & $\omega(\mathrm{Hz})$ & Eigenmode \\
\hline $1^{s t}$ & 249.43 & & 725.19 & & 213.85 & \\
\hline $2^{\text {nd }}$ & 593.33 & & 764.40 & D & 498.67 & - \\
\hline $3^{r d}$ & 851.46 & & 871.53 & $\mathbf{a}=$ & 727.13 & \\
\hline $4^{t h}$ & 1012.27 & 110101 & 1025.57 & $\mathbf{a} \mathbf{a}$ & 819.94 & E \\
\hline
\end{tabular}

Generally, the presence of a fluid field attached to a solid domain diminishes the structure's eigenvalues. Thus, the coupled system has lower natural frequencies, which can be seen in Tab. 1. Also, it is possible to observe that the fluid-structural eigenmodes are composed by a combination of the solid and the fluid eigensolutions. Comparing them, we might conclude that, for this fluid-structure model, the first two coupled eigenmodes are predominantly composed by the $1^{\text {st }}$ and the $2^{\text {nd }}$ structure's eigensolutions. The $3^{\text {rd }}$ and the $4^{\text {th }}$ coupled eigenmodes are predominantly composed by the first two fluid eigensolutions. This analysis is very related to our objective function. It is reasonable that if we chose just the structural field as our design domain, a coupled eigenmode with structural predominance must be chosen as objective function.

For this fluid-structure model, the sensitivity numbers were calculated according to Eq. 26. A sensitivity analysis of the initial design domain was carried out. Figure 2 presents the sensitivity numbers distributions for the design domain's elements for $\omega_{1}$ maximization (a), for both $\omega_{1}$ and $\omega_{2}$ maximization (b) and for $\omega_{1}$ maximization of the structural model without the fluid domain.

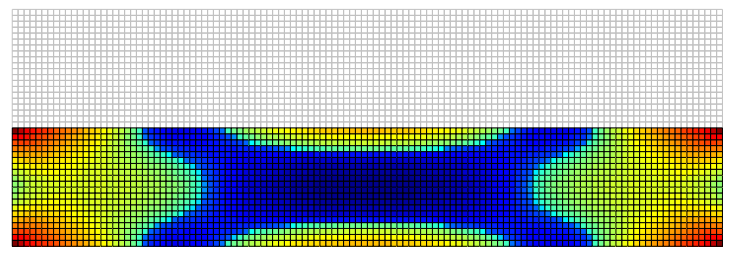

(a)

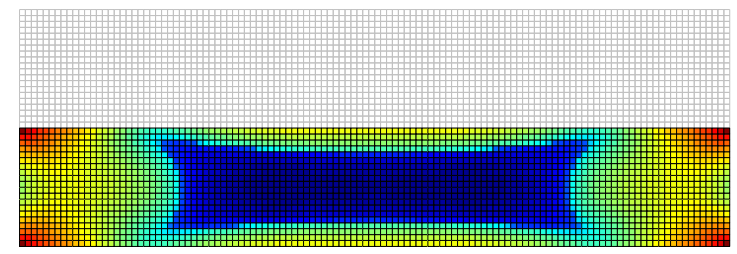

(b)

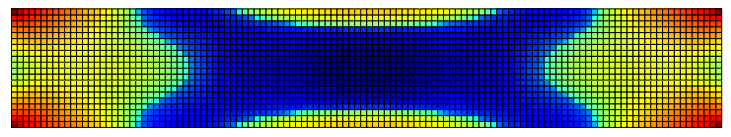

(c)

Figure 2. Sensitivity numbers distributions for: (a) first natural frequency $\omega_{1}$ maximization, (b) both first and second natural frequencies $\omega_{1}$ and $\omega_{2}$ maximization and (c) $\omega_{1}$ maximization for the structural model without a fluid domain. 
These sensitivity numbers indicate the relative elemental efficiency with respect to the objective function. In Fig. 2, the elements with blue color have the lowest sensitivity numbers and those red have the highest numbers. If we want to maximize $\omega_{n}^{2}$, it is reasonable to remove the elements with the lowest sensitivy numbers. It implies an increase in Rayleigh's quotient (Eq. 16), where elements with lower compliance $\left(\Phi_{i}^{T} \mathbf{K} \Phi_{i}\right)$ and higher significative modal mass $\left(\Phi_{i}^{T} \mathbf{M} \Phi_{i}\right)$ are eliminated (void condition) from the finite element model. As expected, the sensitivity numbers for the fluid-structure model only demonstrated symmetry about the vertical-axis, differently from the model without a fluid domain. This is most evident in Fig. 2(b).

For the solid design domain, a bi-directional topology optimization was carried out. BESO started from the initial full design with an evolutionary ratio $E R=1 \%$ until a prescribed volume $V_{f}=80 \%$. The filter radius used was $\mathrm{rmin}=75 \mathrm{~mm}$ and the penalty factor was $p=1.5$. The other parameters were set as $x_{\min }=0.0001, A R_{\max }=5 \%$ and $\tau=0.001$. Figure 3 presents the topologies obtained for two different analysis: (a) for $\omega_{1}$ maximization and (b) for multiple $\omega_{1}$ and $\omega_{2}$ maximization. For these cases, Fig. 4 and 5 presents the evolutionary history of the algorithm.

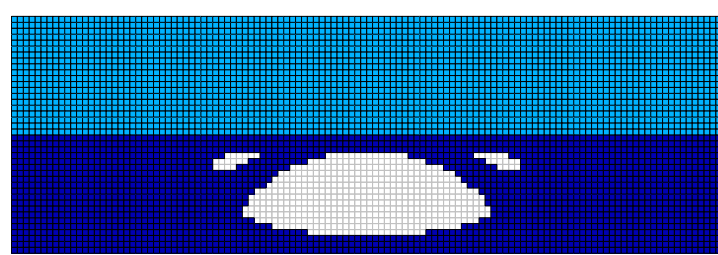

(a)

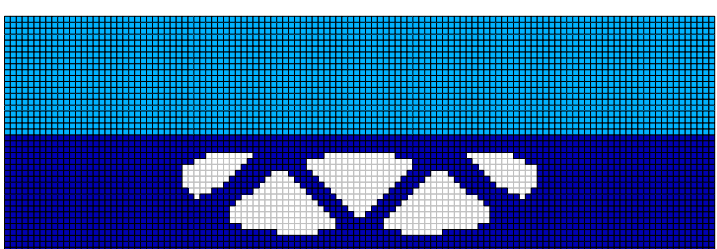

(b)

Figure 3. Topologies with volume constraint $80 \%$ for: (a) first natural frequency $\omega_{1}$ maximization and (b) both first and second natural frequencies $\omega_{1}$ and $\omega_{2}$ maximization.

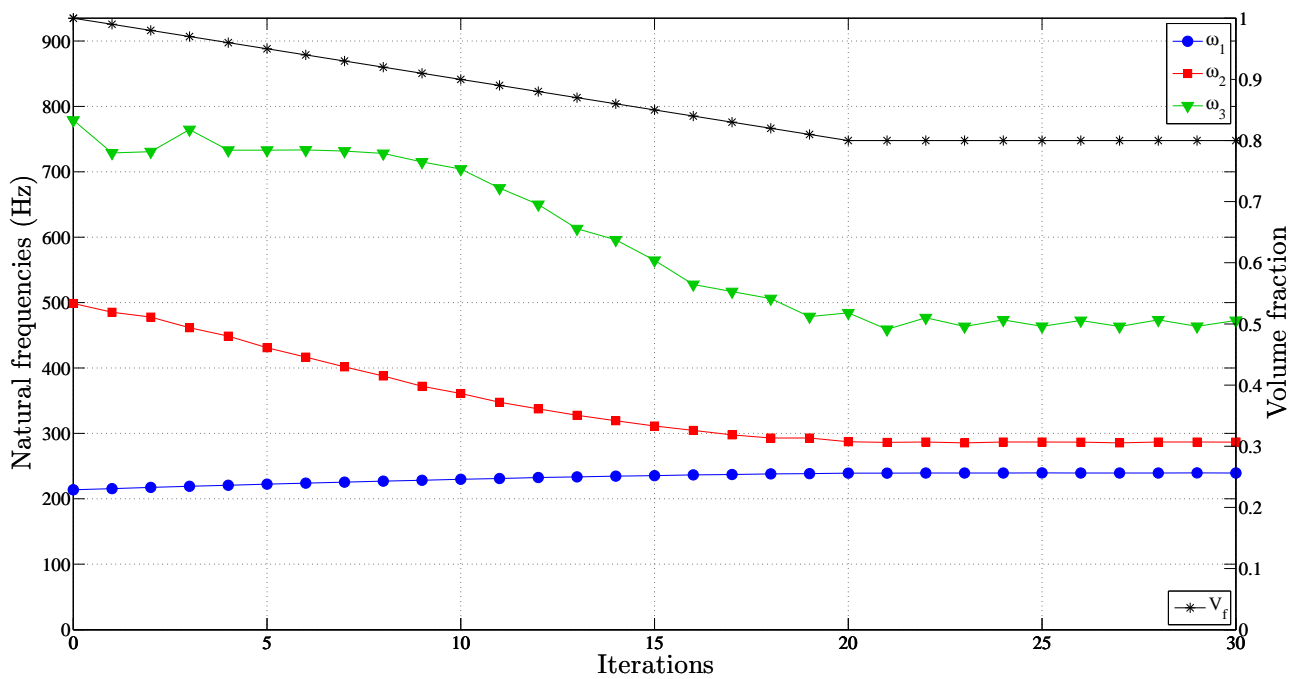

Figure 4. Evolutionary history for $\omega_{1}$ maximization.

In general, for $\omega_{1}$ maximization, the first natural frequency increases and the second and third natural frequencies decrease with material removal. The optimal design (given in 


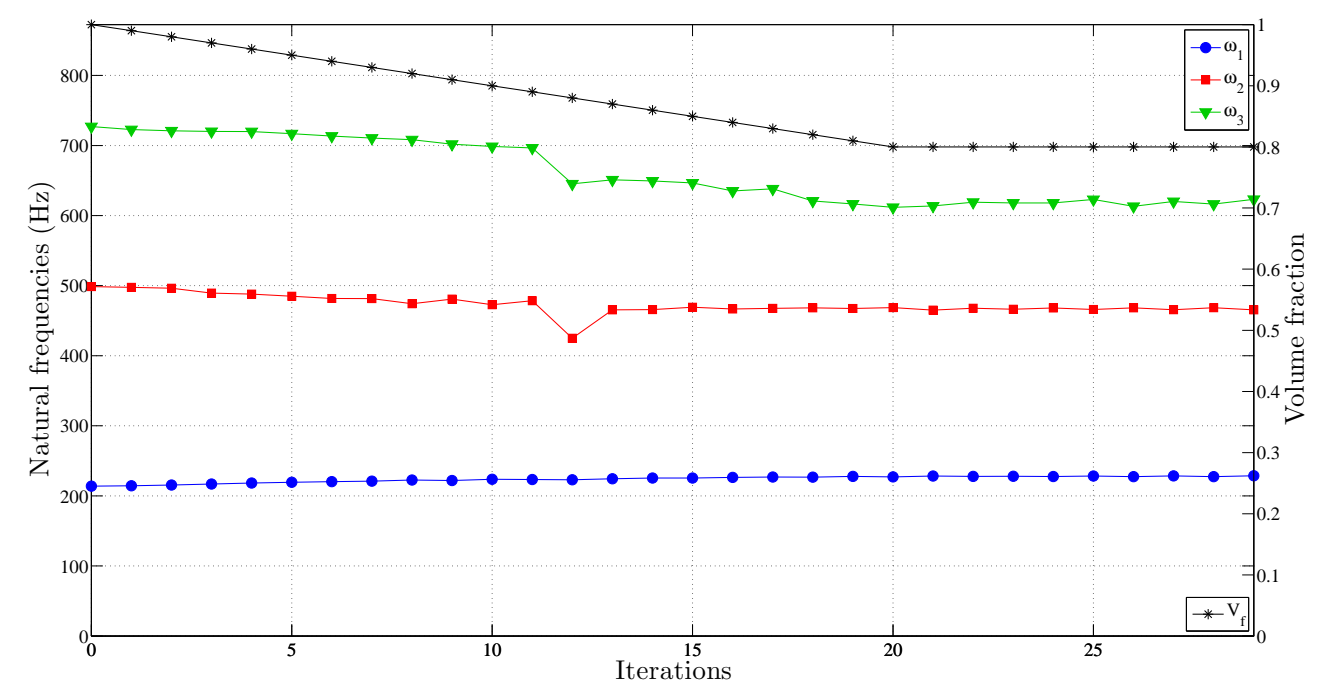

Figure 5. Evolutionary history for both $\omega_{1}$ and $\omega_{2}$ maximization.

Fig. 3(a)) has a fundamental frequency $\omega_{1}=239.36 \mathrm{~Hz}$, which indicates an increase of almost $12 \%$ from the initial full design. It is possible to notice that $\omega_{1}$ is convergent to an almost constant value when the final volume fraction $V_{f}$ is reached. It is also observed that the second natural frequency becomes closer to the first one after some iterations (Fig. 4). It does not happen when the maximization of both multiple $\omega_{1}$ and $\omega_{2}$ is considered, as can bee seen in Fig. 5. In this case, the second natural frequency does not approach to the first one. However, the increase in the fundamental frequency $\omega_{1}$ becomes only $7 \%$. Figure $3(\mathrm{~b})$ presents the topology obtained for this multiple frequencies maximization. The first two eigenmodes of each optimal design case are illustrated in Fig. 6.

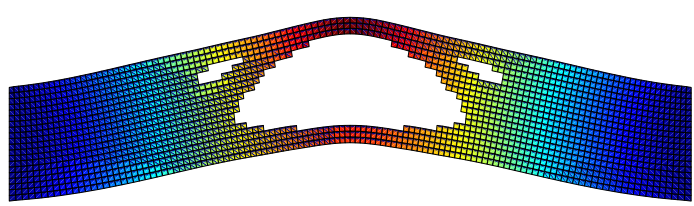

(a)

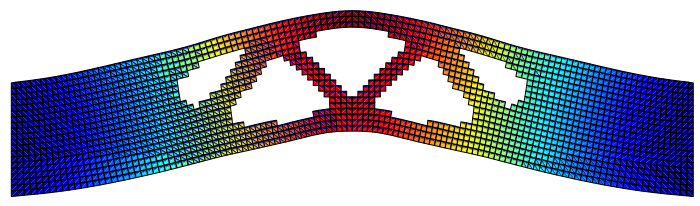

(c)

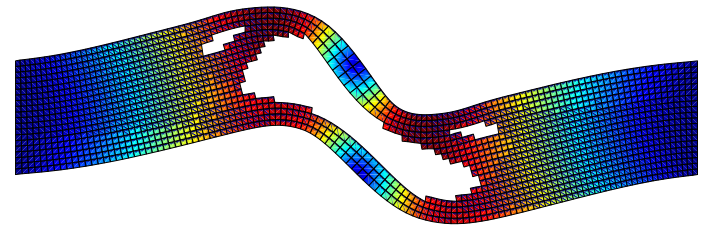

(b)

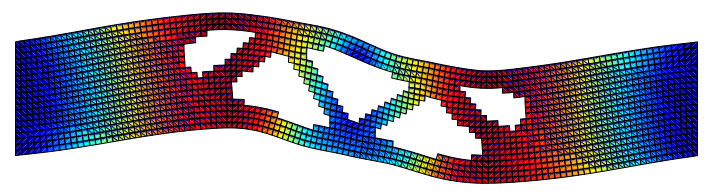

(d)

Figure 6. Eigenmodes for the optimal design cases: (a) first and (b) second eigenmodes for $\omega_{1}$ maximization and (c) first and (d) second eigenmodes for both multiple $\omega_{1}$ and $\omega_{2}$ maximization.

\subsection{Water duct cross section}

In this example, a simplified model of a water duct cross section was chosen. The aim here is to explore a possible application of the BESO method including fluid-structure 
interaction. A material with Young's modulus $E=70 \mathrm{GPa}$, Poisson's ratio $\nu=0.3$ and mass density $\rho_{s}=2700 \mathrm{~kg} / \mathrm{m}^{3}$ must hold a fluid domain with mass density $\rho_{f}=1000 \mathrm{~kg} / \mathrm{m}^{3}$ and speed of sound in the fluid $c=1450 \mathrm{~m} / \mathrm{s}$. This system is under free vibration. The model is illustrated in Fig. 7.

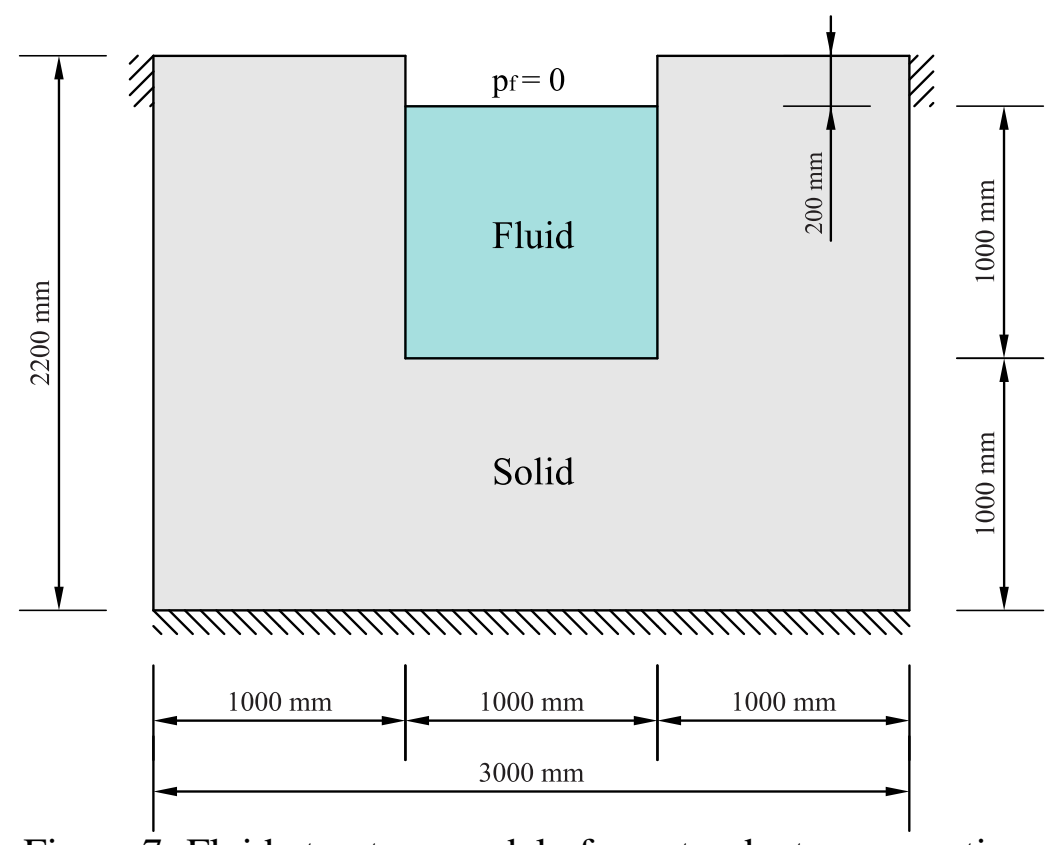

Figure 7. Fluid-structure model of a water duct cross section.

The BESO method was applied with an evolutionary ratio $E R=1 \%$ and a prescribed final volume fraction $V_{f}=50 \%$. The penalty factor used was $p=3$ and the filter radius $r_{\min }=100 \mathrm{~mm}$. The other BESO parameters were $x_{\min }=0.001, A R_{\max }=5 \%$ and $\tau=$ 0.001. The model was discretized with square elements of size $25 \mathrm{~mm}$ and a total number of elements of 10240 . The aim here is to maximizae the fundamental frequency $\omega_{1}$. Figure 8 presents the rank of the sensitivity numbers and the topology obtained for this case.

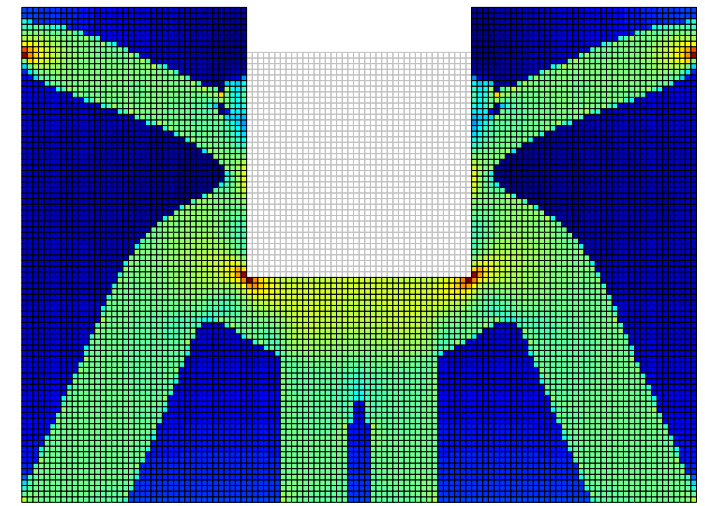

(a)

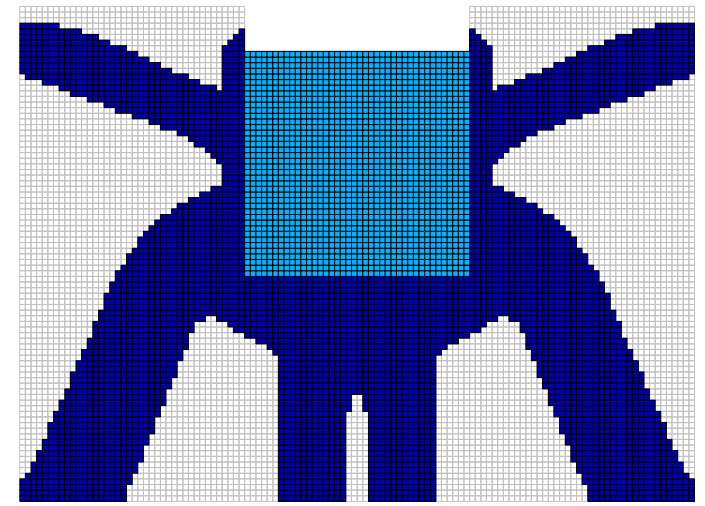

(b)

Figure 8. Sensitivity analysis (a) and topology for fundamental frequency $\omega_{1}$ maximization (b) of a water duct cross section with volue fraction $V_{f}=50 \%$ of the initial design domain.

The optimal design has a fundamental frequency $\omega_{1}=327.29 \mathrm{~Hz}$. Figure 9 presents 
the evolutionary history of the algorithm applied. It is noticed that when a fluid-structure model with a fixed fluid domain is under frequency maximization, the value of the frequency reaches a maximum value and then starts decreasing as showed in Fig. 9. This is directly related with the properties of the solid material and the fluid as well the design volume fraction, where the Rayleigh's quotient is modified each iteration by removing solid elements and keeping the fluid domain fixed.

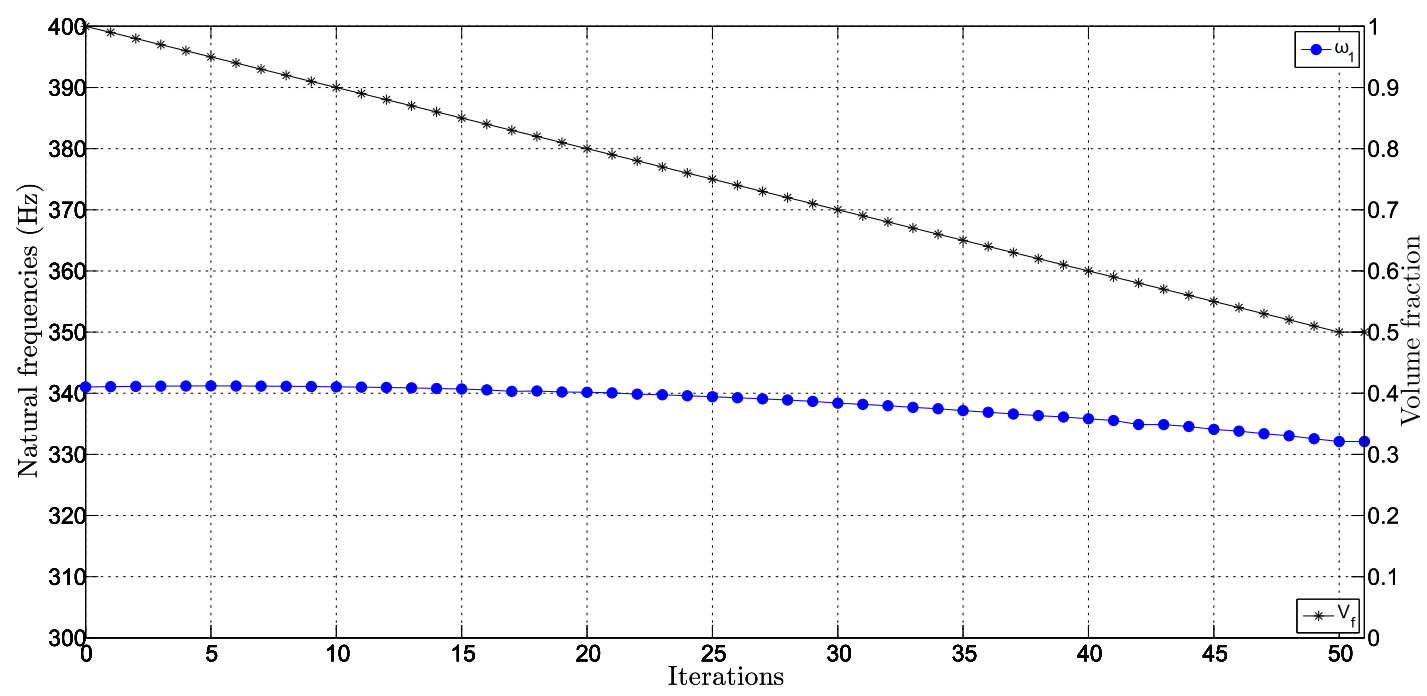

Figure 9. Evolutionary history for $\omega_{1}$ maximization.

\section{Conclusions}

In this paper we have presented the BESO method applied to a class of fluid-structure systems under free vibration. For this kind of problem, the sensitivity numbers were derived. The objective function was the maximization of natural frequencies in structures interacting with a fixed fluid domain. The fluid-structural system's eigensolutions were discussed for both solid and fluid domain separated and for the coupled model as well. It was pointed out that if the design domain is only the solid field, the objective eigenvalue must be correspondent to a fluid-structure eigenvalue with structural predominance. Also, it was noticed that for topology optimization of structures interacting with a fixed fluid domain, the value of the objective frequency reaches a maximum value and then starts decreasing due to relations between the solid material and the fluid as well as the design volume fraction. In general, the BESO method can be applied to this class of problems with good efficiency. For future works, movable interfaces will be considered.

\section{Acknowledgements}

The authors are grateful to CNPq (National Counsel of Technological and Scientific Development) for the financial support of this work. 


\section{REFERENCES}

[1] Bendsoe, M. P. and Sigmund, O., "Generating optimal topologies in structural design". Comput Methods Appl Mech Eng 71, 197-224, 1988.

[2] Bendsoe, M. P. and Sigmund, O., "Topology Optimization - Theory, Methods and Applications”. Berlin, Heidelberg, Springer-Verlag, 2003.

[3] Yoon, G. H., "Topology Optimization for stationary fluid-structure interaction problems using a new monolithic formulation”. Int J Numer Meth Eng 82, 591-616, 2010.

[4] Xu, Z. S., Huang, Q. B. and Zhao, Z. G., "Topology optimization of composite material plate with respect to sound radiation”. Eng Anal Bound Elem 35, 61-67, 2011.

[5] Jang, G. W., Kim, K. J. and Kim, Y. Y., "Integrated topology and shape optimization for compliant MEM's mechanism design". Advanc Eng Soft 39, 1-14, 2010.

[6] Silva, F. I. and Pavanello, R., "Synthesis of porous-acoustic absorbing systems by an evolutionary optimization method". Eng Optimiz 42, 887-905, 2010.

[7] Sigmund, O. and Clausen, P. M., "Topology optimization using a mixed formulation: An alternative way to solve pressure load problems". Comput Methods Appl Mech Eng 196, 1874-1889, 2007.

[8] Maute, K. and Allen, M., "Conceptual design of aeroelastic structures by topology optimization". Struct Multidiscip Optimiz 27, 27-42, 2004.

[9] Bendsoe, M. P. and Sigmund, O., "Material interpolation schemes in topology optimization”. Arch Appl Mech 69, 635-654, 1999.

[10] Hamidian, D, and Seyedpoor, S. M., "Shape optimal design of arch dams using an adaptive neuro-fuzzy inference system and improved particle swarm optimization". Appli Math Model 34, 1574-1585, 2010.

[11] Kreissl, S., Pingen, G, Evgrafov, A. and Maute, K., "Topology optimization of flexible micro-fluidic devices". Struct Multidiscip Optimiz 42, 495-516, 2010.

[12] Xie, Y. M., Steven, G. P., "A simple evolutionary procedure for structural optimization". Comput Struct 49, 885-896, 1993.

[13] Querin, O. M. and Steven, G. P., "Evolutionary structural optimisation (ESO) using a bidirectional algorithm". Eng Comput 15, 1031-1048, 1998.

[14] Huang, X. and Xie, Y. M, "Convergent and mesh-independent solutions for the bidirectional evolutionary structural optimization method". Finite Elem Anal Des 43, 10391049, 2007.

[15] Huang, X. and Xie, Y. M, "Evolutionary topological optimization of vibrating continuum structures for natural frequencies". Comput Struct 88, 357-364, 2010.

[16] Rozvany, G. I. N., "A critical review of established methods of structural topology optimization". Struct Multidiscip Optimiz 37, 217-237, 2009.

[17] Huang, X. and Xie, Y. M, "A further review of ESO type methods for topology optimization”. Comput Struct 41, 671-683, 2010.

[18] Xie, Y. and Huang, X., "Evolutionary Topology Optimzation of Continuum Structures: Methods and Applications". West Sussex, John Wiley Sons, 1st edition, 2010.

[19] Davidsson, P., "Structure-acoustic analysis; Finite element modelling and reduction methods". Doctoral Thesis. Lund University, Lund, Sweden, 2004. 
[20] Pedersen, N. L., "Maximization of eigenvalues using topology optimization". Struct Multidiscip Optimiz 20, 2-11, 2000.

[21] Huang, X. and Xie, Y. M., "Bi-directional evolutionary topology optimization of continuum structures with one or multiple materials". Comput Mech 43, 393-401, 2009.

[22] Sigmund, O. and Peterson, J., "Numerical instabilities in topology optimization: A survey on procedures dealing with checkerboards, mesh-dependencies and local minima". Struct Optimiz 16, 68-75, 1998. 